Ryland 11), with a poem by Kepler. This contribution sets higher standards of scholarship than some of the astronomical items. The short papers on Kepler's wanderings from Württemberg to Graz, to Prague, and so on, would make on their own a most enmanners no less than to the places of Kepler's time.

One of the most surprising things about the 1971 symposia is that so few historians took the opportunity of adding to our understanding of Kepler's optics. A précis of J. A. Lohne's study of Kepler, Harriot, and the sine law is worth noticing, and yet for the full text one must turn to the Weil der Stadt proceedings. That a similar remark must be made for many of the articles in this volume is not a weakness but a source of strength. This is joyable Baedekker's guide to the

essentially a 'State of the Art' report. In a report within a report, Robert $S$. Westman considers two early historiographical traditions in Kepler scholarship. In one of these, the history of science was regarded as a useful repository of still valued scientific ideas. The second, hermeneutical, tradition entailed an investigation of the philosophical foundations of Kepler's dis. coveries. Westman, speaking in Phil. adelphia towards the end of 1971, placed those papers given to the European conferences against this double historiographical background. This impressive and important volume, in an almost uniformly important series, is therefore complete to a fault, containing as it does its own review. It will be consulted long after its weighty $(3 \mathrm{~kg})$ contents have parted company with its covers.

J. D. North

\section{Applying a technique}

Mössbauer Spectroscopy. (Topics in Applied Physics, Vol. 5.) Edited by U. Gonser. Pp. xviii +241 . (Springer : Berlin and New York, 1975.) DM70; $\$ 30,10$.

THE increasing use of the Mössbauer technique during the past 15 years in fields as diverse as relativity and archaeology, resulted in the publication of about 1,200 research papers in 1974 . Its use in conjunction with more standard techniques in, for instance, chemistry and metallurgy departments means that it is no longer practicable to produce a book giving a critical study of the whole range of applications. Several good introductions to the basic principles exist already but the purpose of the present volume is both to introduce the technique to nonspecialists and also to present them with general information on the applications related to their own fields of interest. Thus the introductory chapter is followed by five chapters, written by different authors, concerned with applications in chemistry, magnetism, biology, lunar geology and mineralogy and physical metallurgy. The level seems to be aimed at that of the research worker although the treatment varies from chapter to chapter, with those conccrned with chemistry and magnetism requiring familiarity with the methods of quantum mechanics.

In the introduction, the conditions required for the emission of Mössbauer $\gamma$ rays are described together with the properties of the hyperfine interactions, between the nucleus and its electronic environment, which form the basis of a Mössbauer measurement. A short experimental section is marred by poor quality spectra and a misleading comparison of $\gamma$-ray detectors. Two chapters are devoted to a discussion of the information obtained from the hyperfine interactions, concerning the electronic structure and bonding properties in chemical compounds in general, and in metal proteins. In the latter case the potentially valuable use of the technique to determine the state of the iron in clinical specimens of blood or tissue is mentioned, with the hope that this could lead to advances in medical diagnosis. In the chapter on concerned largely with studies of spin orientations in mixed iron oxides. That chapter, particularly, suffers somewhat from being restricted to the isotope iron-57.

The use of the Mössbauer effect as an analytical tool to determine molecular composition is brought out in the chapter on lunar geology and mineralogy, though little discussion is given of the main findings of other measurements. The final chapter on physical metallurgy seems to be at about the right level for an introductory survey. Methods are given for the analysis of the spectra of alloys and intermetallic compounds, with many examples including the study of carbon atoms in steels, the invar problem and the structure of amorphous alloys.

Although the level of presentation varies, in general it is higher than that usually found in an introduction for the non-specialist. As such, the book is more likely to be read by the Mössbauer specialist looking for a presentation of applications outside his own field of study. More comprehensive surveys in each field exist already and the price of $\$ 30.10$ is rather high for the purchase of a personal copy.

G. Longworth magnetism there is no mention of work on metals and alloys, and the text is

\section{Finite geometries and designs}

Graph Theory, Coding Theory and Block Designs. (London Mathematical Society Lecture Note Series, No. 19.) By P. J. Cameron and J. H. van Lint. Pp. $v+114$. (Cambridge University, Cambridge, London and New York, September 1975.) £2.80.

THIS splicing of lecture notes from two courses of seminar lectures given at Westfield College in 1973 is intended for a specialised group of mathematicians, combinatorialists interested in finite geometries and designs, and serves to introduce them to those parts of graph theory and algebraic coding theory that have recently interacted fruitfully with design theory. The authors have, however, provided a brief introduction to design theory proving certain elementary results, stating certain deeper results, and describing many of those designs that arise geometrically. Thus, in principle, the book can be read by any mathematician.

Chapters 2-5 are concerned with the connections between design theory and strongly regular graphs. Chapter 6 is a brief survey of known results concerning so-called "two graphs", a combinatorial structure that arises naturally in the study of doubly-transitive permutation groups. The remaining nine chapters are devoted to those aspects of algebraic coding theory of interest to design theorists. Chapters 9-11 constitute an introduction to coding theory and chapter 15, the last, an introduction to association schemes (a generalisation of strongly regular graphs) and some of the recent work of Delsarte. Sandwiched between are five chapters concerned with those codes of greatest combinatorial interest: Reed-Muller codes, self-orthogonal codes, quadratic-residue codes, symmetry codes, nearly perfect and uniformly packed codes.

The expert will note that proposition 13.4 is incorrect without an added hypothesis and that the remark on page 69 is false since ovals are always seen in the dual. In spite of minor flaws and sometimes sketchy proofs this slender volume should prove useful to its intended audience.

E. F. Assmus, Jr

Erratum. The size of the Mayan bas relief shown on p.349 of the reviews section (Nature, 259, January 29. 1976) was incorrectly stated as $1.3 \times 1.3 \mathrm{~cm}$. The correct size is $1.3 \times 1.3 \mathrm{~m}$. 